Objectives: To evaluate the quantitative statuses of peripheral CD4 ${ }^{+} T$ subpopulations in patients with gout and further investigate the effects of immunomodulatory combination therapies on those cells.

Methods: Total 247 patients who met the clinical criteria of gout from the American College of Rheumatology and 206 healthy controls (HCs) were enrolled in this retrospective cross-sectional study. Among those patients, 70 follow-up patients donated their peripheral blood after receiving immunomodulatory drugs (e.g., low-dose interleukin-2, rapamycin, metformin, retinoic acid, etc). The absolute numbers of Th1, Th2, Th17 and Tregs in peripheral $C D 4^{+} T$ subsets were detected by flow cytometry combined with standard absolute counting beads.

Results: Compared with HCs, the absolute numbers of Th1 and Th17 were evidently increased in gout patients $(P<0.001)$, while the level of Tregs was significantly decreased $(P<0.05)$ (Figure 1$)$. After immunomodulatory combination treatments, there were dramatical increases in a wide variety of $\mathrm{CD} 4^{+} T$ subsets such as Th1, Th17 and Tregs $(P<0.05)$. Interestingly, the increased amount of Tregs was much more than that of other Teffs, leading to the decrease ratios of Teffs/Tregs such as Th2/Tregs, restoring immune homeostasis (Figure 2).

Conclusion: This cross-sectional study clarified the abnormal statuses of $\mathrm{CD} 4^{+} \mathrm{T}$ subsets in gout patients, suggesting that CD4+T subsets, especially Tregs, might be relevant and play a crucial role in the pathogenesis of gout, thus providing a potential therapeutic target for gout patients. Immunomodulatory combination therapies effectively increase the number of Tregs and may help for gout patients' symptom remission.
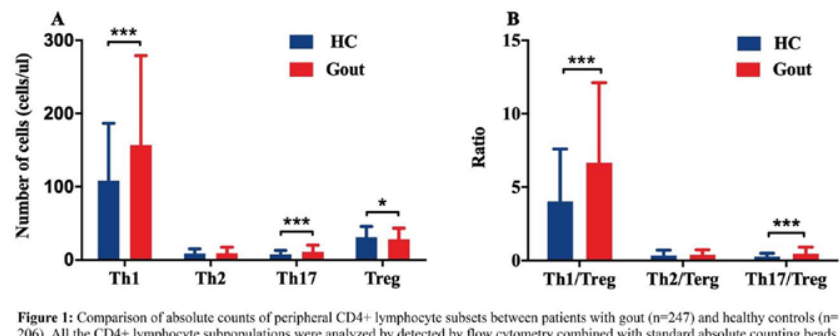
206). All the CD4+ lymphocyle subpopulations were analyzed by detected by flow cylometry combined with standard absolute counting bead.
Data were presented as mean $\mathrm{SD}$ and statistical analysis was delermined by two-tailed unpaired t-test. Paticnts had lower levels of Tregs and

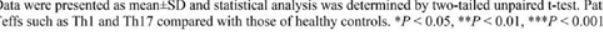
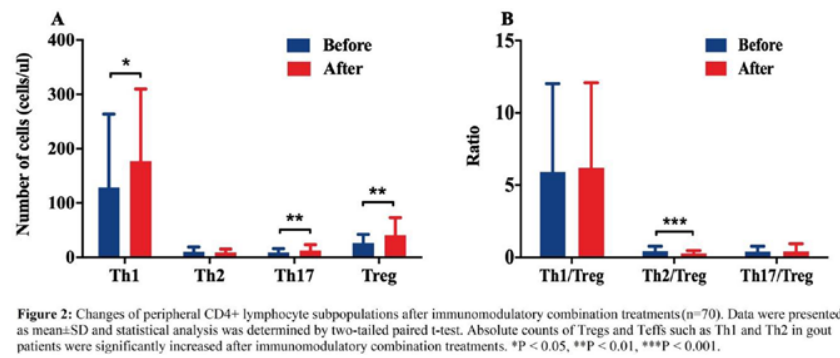

References:

[1] Ramsubeik K, Ramrattan LA, Kaeley GS, et al. Effectiveness of healthcare educational and behavioral interventions to improve gout outcomes: a systematic review and meta-analysis. Therapeutic advances in musculoskeletal disease 2018;10(12):235-52. doi: 10.1177/1759720x18807117 [published Online First: 2018/12/06]

[2] Stiburkova B, Pavelcova K, Pavlikova M, et al. The impact of dysfunctional variants of ABCG2 on hyperuricemia and gout in pediatric-onset patients. Arthritis Res Ther 2019;21(1):77. doi: 10.1186/s13075-019-1860-8 [published Online First: 2019/03/22]

[3] Raucci F, Iqbal AJ, Saviano A, et al. In-depth immunophenotyping data relating to IL-17Ab modulation of circulating Treg/Th17 cells and of in situ infiltrated inflammatory monocytes in the onset of gouty inflammation. Data Brief 2019;25:104381. doi: 10.1016/j.dib.2019.104381 [published Online First: 2019/09/07]

Acknowledgments: None.

Disclosure of Interests: None declared

DOI: 10.1136/annrheumdis-2020-eular.2110

\section{AB0033 \\ CHARACTERIZATION OF THE PERIPHERAL B CELL COMPARTMENT IN PATIENTS WITH EOSINOPHILIC GRANULOMATOSIS WITH POLYANGIITIS}

R. Lorenzetti ${ }^{1}$, M. Engesser ${ }^{1}$, R. Voll ${ }^{1}$, A. Troilo ${ }^{1}$, I. Janowska ${ }^{1}$, M. Rizzi ${ }^{1}$, N. Venhoff ${ }^{1}$, J. Thiel ${ }^{1}{ }^{1}$ Vasculitis Center Freiburg, Department of Rheumatology and Clinical Immunology, University Medical Center Freiburg, Freiburg, Germany

Background: Eosinophilic granulomatosis with polyangiitis (EGPA) is a rare form of systemic vasculitis, which is characterized by bronchial asthma hypereosinophilia, and systemic vasculitis. B-lymphocytes play a key role in EGPA as producers of $\mathrm{IgE}$ and anti-neutrophil cytoplasmic antibodies (ANCAs). Indeed, the neutrophils that are targeted by these antibodies are widely described as the mechanism of endothelial damage in this disease. On the other side, the therapeutic response to rituximab in EGPA patients provides evidence for a role of B-cells in the pathogenesis of EGPA. Therefore characterizing B cell subpopulations may help in understanding the disease and the treatment.

Objectives: To characterize the peripheral B cell compartment in patients with EGPA and to analyze the in vivo potential of B lymphocytes to class-switch to $\mathrm{IgE}$ and to assess in vitro the differentiation potential of naïve B cells of EGPA patients into IgE-secreting plasmablasts.

Methods: Clinical characteristics of the patients, including organ involvement and treatment regimen were evaluated. Laboratory work-up included ANCA-status, eosinophils, IgE, IgG, IgA, IgM, and peripheral CD19+ B-cell count. For immunophenotyping isolated PBMCs were stained with monoclonal or polyclonal antibodies and $B$ cells were classified into: naïve, marginal zone, class-switched memory B cells, unconventional memory $B$ cells, transitional and plasmablasts. Furthermore, the expression of $\lg \mathrm{G}+$ and subclasses $\lg \mathrm{g} 1-4, \lg \mathrm{A}^{+}, \lg \mathrm{E}^{+} \mathrm{B}$ cells, BAFFR and TACl was quantified. For in vitro differentiation assays magnetically isolated $B$ lymphocytes from EGPA patients and age-matched healthy controls were stimulated with CD40L, IL-21 and IL-4. Starting the culture with equal number of B cells, the absolute number of plasmablasts, and IgE class switched cells after 9 days was determined by counting the events in the CD27highCD38high gate or the $\operatorname{lgG} / \mathrm{A} / \mathrm{D}-\mathrm{IgE}^{+}$ gate by flow cytometry. IgE secretion in the supernatant was measured by ELISA. JAK-STAT signalling pathway was analyzed in response to IL-4 and IL-21 stimulation and phosphorylation of STAT5 and 6 measured by flow cytometry.

Results: 34 patients with EGPA diagnosed according to ACR and $\mathrm{CHC}$-criteria were included into the study. Ten of these patients were analysed separately because they received rituximab therapy. Peripheral $B$ cell numbers in EGPA patients were markedly diminished. B cell subpopulation phenotyping showed in average $57.9 \%$ naïve B cells, $12.5 \%$ marginal zone like B cells and $19.2 \%$ switched memory B cells. Plasmablasts constituted in average $1.15 \%$ of the peripheral B cell compartment, transitional B cells $2.0 \%$. Interestingly, the expression of BAFF receptor and $\mathrm{TACl}$ in the memory $\mathrm{B}$ cell subset was significantly decreased in EGPA patients when compared with healthy donors. In vitro assays of isolated B cells from EGPA patients demonstrated an increased proportion of IgE-class-switched B cells after 9 days of culture under IL4 stimulation compared with controls. However, no differences were observed in the phosphorylation of STAT5 and STAT6 after stimulation with IL-4 or IL-21.

Conclusion: In the EGPA-patients we observed markedly diminished B-cells despite of normal lymphocyte counts. $B$ cells showed a reduced expression of BAFF-R and TACI. Class switch to IgE is enhanced in EGPA patients.

Disclosure of Interests: None declared

DOI: 10.1136/annrheumdis-2020-eular.5944

\begin{tabular}{|l|l|}
\hline AB0034 & EARLY IMMUNIZATION AGAINST TREATMENT IS \\
ASSOCIATED TO POOR CLINICAL RESPONSE AT 6 \\
MONTHS AND LOW NUMBER OF TRANSITIONAL B \\
CELLS AT BASELINE IN RHEUMATOID ARTHRITIS \\
PATIENTS TREATED BY ADALIMUMAB
\end{tabular}

C. Lucas ${ }^{1}$, S. Rodriguez ${ }^{2}$, R. Jean ${ }^{2}$, J. D. Albert ${ }^{1}$, G. Coiffier ${ }^{1}$, E. Dumontet ${ }^{2}$, K. Tarte ${ }^{2}$, P. Amé-Thomas ${ }^{2}$, A. Perdriger ${ }^{1} .{ }^{1}$ University Hospital, Rheumatology, Rennes, France; ${ }^{2}$ INSERM1236, Rennes, France

Background: Circulating anti-drug antibodies (ADAs) are detectable approximatively in $33 \%$ of adalimumab treated rheumatoid arthritis (RA) patients, often within the first 6 months of therapy ${ }^{1-2}$. Classically, circulating ADAs associate with their specific drugs to form immune complexes, increasing drug clearance, and by this mechanism reducing therapeutic effect ${ }^{3}$. B cell involvement leading to ADAs production is not yet well established. 
Objectives: To study early ADAs formation according to clinical response to an adalimumab therapy in RA patients and the relationship between ADAs and circulating $B$ cell subsets.

Methods: 28 RA patients and 13 healthy controls were included. Patients all presented inadequately controlled RA under conventional treatment, were naive of biotherapies, and started an adalimumab treatment at baseline (M0). Responder status was determined according to the DAS28CRP score $(<0$ or $>3.2)$ at 3 (M3) and 6 months (M6). ADAs plasma concentration $>10 \mathrm{pg} / \mathrm{mL}$ at $\mathrm{M} 3$ defined the immunized patient group. Circulating B cell subsets were quantified by flow cytometry at $\mathrm{M} 0$ and $\mathrm{M} 3$.

Results: 11 (42.3\%) patients were immunized at M3. Among them, 4 (36.4\%) were responders at M6 and 7 (63.6\%) were non-responders. Presence and concentration of ADAs at M3 was associated to non-responder status at M6 $(p=0.043 ; p=0.042)$. Immunized patients had lower transitional $B$ cells count at M0 compared to non-immunized patients $(p=0.031)$.

Conclusion: A high but classical proportion of RA patients developed ADAs after only 3 months of adalimumab treatment. This immunization was associated to non-responder status at $\mathrm{M} 6$ and to a low blood transitional B cells count at baseline. Our results suggest transitional B cells implication in

RA activity and biotherapy resistance due to immunization. Low concentrations of transitional $B$ cells could be an early biomarker of immunization process against adalimumab.

References:

[1] Bartelds GM et al. JAMA. 2011

[2] Moots RJ et al. PLoS ONE. 2017

[3] Van Schouwenburg PA et al. Ann Rheum Dis. 2013

Table. Patients characteristics at baseline

\begin{tabular}{lccc}
\hline Characteristics & $\begin{array}{c}\text { All RA patients } \\
(\mathbf{n}=\mathbf{2 8})\end{array}$ & $\begin{array}{c}\text { M6 responders } \\
(\mathbf{n}=16)\end{array}$ & $\begin{array}{c}\text { M6 } \\
\text { non-responders } \\
(\mathbf{n}=10)\end{array}$ \\
\hline Age (years) & $60.5[47-78]$ & $65.5[47-76]$ & $54[47-78]$ \\
Sex ratio M/F (\% of F) & $0.4(71,4 \%)$ & $0.5(68.8 \%)$ & $0.3(80.0 \%)$ \\
Disease duration, (years) & $5.6[0.7-43.0]$ & $6.8[1.0-43.0]$ & $2.9[0.7-31.0]$ \\
Oral steroid use, (\%) & $18(64,3 \%)$ & $9(56.2 \%)$ & $8(80.0 \%)$ \\
Oral steroids, dose (mg/day) & $5.0[2.0-15]$ & $5.0[4.0-12.5]$ & $8.5[2.5-15.0]$ \\
Methotrexate use, (\%) & $24(50.9 \%)$ & $14(87.5 \%)$ & $8(80.0 \%)$ \\
Methotrexate, dose (mg/week) & $20[10-25]$ & $20[10-25]$ & $15[10-20]^{*}$ \\
Leflunomide use, (\%) & $3(10.7)$ & $1(6.3 \%)$ & $2(20.0 \%)$ \\
Leflunomide, dose (mg/day) & $20[20-20]$ & $20[20-20]$ & $20[20-20]$ \\
CRP, (mg/dL) & $5.5[1.0-57.0]$ & $6.6[1.0-46.8]$ & $3.6[1.0-57.0]$ \\
DAS28CRP score & $4.3[3.3-5.7]$ & $4.1[3.3-5.2]$ & $4.5[3.4-5.7]$ \\
RF positive, (\%) & $20(71.4 \%)$ & $12(75.0 \%)$ & $6(60.0 \%)$ \\
RF, (U/mL) & $116[19-640]$ & $88[21-640]$ & $181[19-336]$ \\
ACPA positive, (\%) & $25(89.3 \%)$ & $15(93.8 \%)$ & $8(80.0 \%)$ \\
ACPA, (U/mL) & $340[11-340]$ & $340[14-340]$ & $340[11-340]$ \\
\hline
\end{tabular}

Values are medians with ranges and frequencies with percentages. ${ }^{*} \mathrm{p}=0.050$

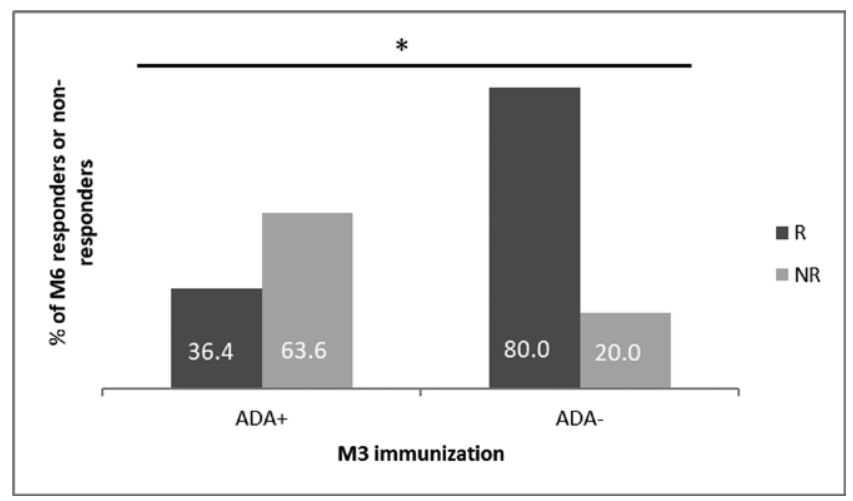

Figure 1. Graph 1 Immunization against treatment at 3 months and clinical response at 6 months in RA patients $(n=26)$. Presence of ADAs at 3 months is associated to non-responder status at 6 months. Fisher exact test.R, responders at 6 months; NR, non-responders at 6 months; ADA+, immunized patients at 3 months; ADA-, non-immunized patients at 3 months.

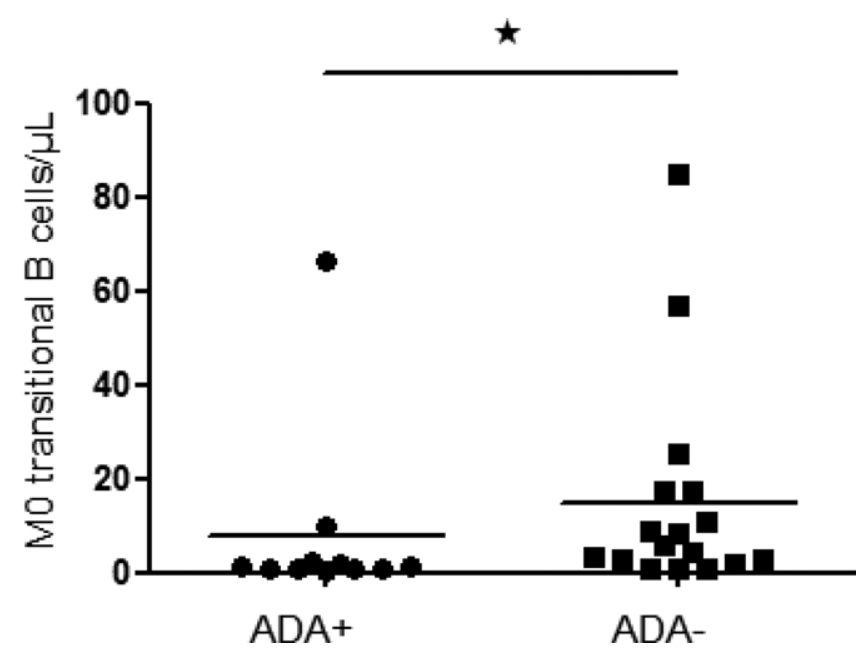

Figure 2. Graph 2 Absolute number of transitional $B$ cells at baseline in RA patients $(n=28)$ according to immunized status at 3 months. Immunized patients at 3 months had lower transitional $B$ cells at baseline than non-immunized patients. ADA+, immunized patients at 3 months; ADA-, non-immunized patients at 3 months. Data represent the mean; ${ }^{*} p<0.05$ by Mann-Whitney $U$ test.

Disclosure of Interests: None declared

DOI: 10.1136/annrheumdis-2020-eular.3938

\section{$\mathrm{AB} 0035$ \\ TWO DIFFERENT ABNORMAL BEHAVIORS IN CD4+T LYMPHOCYTES IN FIBROMYALGIA PATIENTS AND FIBROMYALGIA ASSOCIATED TO SJÖGREN'S SYNDROME}

J. Monserratsanz ${ }^{1}$, A. Movasat ${ }^{2}$, M. D. Sosa Reina ${ }^{1}$, A. M. Gomez

Lahoz $^{1}$, C. Bohórquez ${ }^{1}$, A. Pérez Gómez ${ }^{2}$, L. Ruiz², M. J. Cabezas Leon², A. Sánchez Atrio ${ }^{2}$, F. Albarrán ${ }^{2}$, M. Álvarez de Mon ${ }^{3} .{ }^{1}$ Universidad de Alcala. Facultad de Medicina, Medicina y Especialidades Médicas, Alcala de Henares, Spain; ${ }^{2}$ Hospital Príncipe de Asturias, Reumatología, Alcala de Henares, Spain; ${ }^{3}$ Hospital Príncipe de Asturias. Universidad de Alcala, Medicina y Especialidades Médicas, Alcala de Henares, Spain

Background: Primary fibromyalgia syndrome is a prevalent rheumatic condition characterized by widespread pain and whose etiopathogenesis is not well understood. Fibromyalgia can also be secondary to other rheumatic diseases like Sjogren's syndrome; however, its relation to this disease is unknown. It has been suggested that the immune system is involved in their pathogenesis. The role of activation stages and cytokines profiles of CD4+T lymphocytes in fibromyalgia or fibromyalgia secondary to Sjogren's syndrome are completely unclear and could play a key role in the pathophysiology of these diseases.

Objectives: The objective of this study is to investigate the counts and distribu tion of the CD4+T lymphocyte activation subsets and their pattern of cytokine production in women with primary fibromyalgia, fibromyalgia secondary to Sjogren's, Sjogren's syndrome and healthy controls $(\mathrm{HC})$. The counts and distribution of naïve $\left(T_{N}\right)$, central memory $\left(T_{C M}\right)$, effector memory $\left(T_{E M}\right)$ and effector $\left(T_{E}\right)$ CD4+T lymphocyte subsets were analyzed in these diseases. Furthermore, we investigated their pattern of IL-4, IL-10, IL-17A, IFNץ, and TNFa production. Methods: Counts and distribution of CD4+T subsets $\left(T_{N}, T_{C M}, T_{E M}, T_{E)}\right.$ and their cytokine producing capacity were measured using multiparametric flow cytometry in peripheral blood mononuclear cells (PBMC) from 20 primary fibromyalgia, 15 fibromyalgia associated to Sjögren and 15 primary Sjögren patients and 15 female controls. Fibromyalgia and/or Sjögren's syndrome were diagnosed based on ACR criteria. CD4+T cell activation stages were analyzed by the expression of the CD3, CD4, CD45RA, CD27 and CCR7 antigens. Cytokine CD4+T producing cells subsets were assayed stimulating PBMC during 6 hours, fixed, permeabilized and simultaneously stained with IL-4, IL-10, IL-17A, IFN $\gamma$, and TNFa intracellular cytokines.

Results: Fibromyalgia patients showed a significant increase in the CD4+T, $T_{N}$ and $T_{C M}$ cells counts with compared to fibromyalgia secondary to Sjogren, Sjogren's syndrome and HC. The counts of IL-17A, IL-4 and IFNY producing 